Review Article

\title{
Glu504Lys Single Nucleotide Polymorphism of Aldehyde Dehydrogenase 2 Gene and the Risk of Human Diseases
}

\author{
Yan Zhao ${ }^{1}$ and Chuancai Wang ${ }^{2}$ \\ ${ }^{1}$ Department of Bioengineering, Harbin Institute of Technology at Weihai, Shandong 264209, China \\ ${ }^{2}$ Department of Mathematics, Harbin Institute of Technology at Weihai, Shandong 264209, China \\ Correspondence should be addressed to Yan Zhao; zhaoyan@hitwh.edu.cn and Chuancai Wang; wangcc01@hitwh.edu.cn
}

Received 7 May 2015; Revised 29 July 2015; Accepted 19 August 2015

Academic Editor: Jing Zhang

Copyright (c) 2015 Y. Zhao and C. Wang. This is an open access article distributed under the Creative Commons Attribution License, which permits unrestricted use, distribution, and reproduction in any medium, provided the original work is properly cited.

\begin{abstract}
Aldehyde dehydrogenase (ALDH) 2 is a mitochondrial enzyme that is known for its important role in oxidation and detoxification of ethanol metabolite acetaldehyde. ALDH2 also metabolizes other reactive aldehydes such as 4-hydroxy-2-nonenal and acrolein. The Glu504Lys single nucleotide polymorphism (SNP) of ALDH2 gene, which is found in approximately $40 \%$ of the East Asian populations, causes defect in the enzyme activity of ALDH2, leading to alterations in acetaldehyde metabolism and alcohol-induced "flushing" syndrome. Evidence suggests that ALDH2 Glu504Lys SNP is a potential candidate genetic risk factor for a variety of chronic diseases such as cardiovascular disease, cancer, and late-onset Alzheimer's disease. In addition, the association between ALDH2 Glu504Lys SNP and the development of these chronic diseases appears to be affected by the interaction between the SNP and lifestyle factors such as alcohol consumption as well as by the presence of other genetic variations.
\end{abstract}

\section{Introduction}

Aldehyde dehydrogenase (ALDH) 2 is a mitochondrial enzyme that catalyzes the oxidation of acetaldehyde, an intermediate of ethanol metabolism [1]. It is also important in metabolizing other toxic aldehydes such as 4-hydroxy2-nonenal (4-HNE) and acrolein [2]. The Glu504Lys single nucleotide polymorphism (SNP) of $A L D H 2$ gene, which occurs with an incidence of $35-57 \%$ in different East Asian subpopulations, causes defect in the enzyme activity of ALDH2, leading to alterations in acetaldehyde metabolism and markedly reduced alcohol tolerance $[3,4]$. Epidemiological studies have linked ALDH2 Glu504Lys SNP with increased risk for human diseases including cardiovascular disease (CVD), cancer, and late-onset Alzheimer's disease (AD) [5-8]. The association between ALDH2 Glu504Lys SNP and the development of these diseases is also related to the effect of the SNP on lifestyle factors such as alcohol consumption and its interaction with other genetic variations.

\section{ALDH2 in Ethanol Metabolism and Beyond}

ALDH2 is a member of $\mathrm{NAD}(\mathrm{P})^{+}$-dependent ALDH supergene family that catalyzes the oxidation of endogenous and exogenous aldehydes to their corresponding carboxylic acids (reviewed in [9]). The enzyme activities of ALDHs mediate the formation of molecules with important biophysiological functions such as retinoic acid, betaine, and gammaaminobutyric acid [10-12]. On the other hand, aldehydes are highly reactive compounds, which can form adducts with proteins, DNA, and lipids, affecting the function of these biomolecules and leading to cell toxicity. Endogenous aldehydes are generated during the metabolism of amino acids, carbohydrates, lipids, and vitamins as well as the biotransformation of many drugs and environmental chemicals [10, 1315]. Meanwhile, aldehydes are present in the environment and in our foods. Indeed, aldehydes can be produced in high concentrations by heating fats and sugars (reviewed in [16]). Thus, the detoxification of harmful aldehydes generated endogenously or ingested from environment and foods is an important role of ALDHs. 
ALDH2 is best known for its ability to oxidize acetaldehyde, an intermediate of ethanol metabolism. Animal studies have shown that ALDH2 is a major enzyme for acetaldehyde metabolism [1]. In addition, ALDH2 is important for the detoxification of reactive aldehydes such as 4-HNE and acrolein [2]. 4-HNE is an $\alpha, \beta$-unsaturated aldehyde formed during lipid peroxidation in vivo [17]. Acrolein is found in small amounts in many foods such as cheese, fish, bread, and alcoholic beverages, while high levels of acrolein can be detected in cigarette smoke and overheated oils (reviewed in [18]). ALDH2 may be one of the essential mechanisms for the removal of these reactive aldehydes and protecting cells and organs from these toxic aldehydes. Furthermore, it has been suggested that ALDH2 may have more than one catalytic function. For example, ALDH2 can act as nitrate reductase, which catalyses the formation of 1,2-glyceryl dinitrate and nitrite from nitroglycerin, leading to the production of cGMP and vasorelaxation [19].

\section{SNP in $A L D H 2$ Gene}

Human ALDH2 gene is found on chromosome 12 (12q24.2) and encodes a protein localized in mitochondria matrix. The enzyme is expressed abundantly in liver and is also present in heart, kidney, muscle, and brain [20]. Analyses of the protein structure of ALDH2 have revealed that the enzyme is a tetramer of four identical subunits, each of which is composed of three main domains: the catalytic domain, the $\mathrm{NAD}^{+}$-binding coenzyme domain, and the oligomerization domain. A dominant-negative ALDH2 SNP, which occurs with an incidence of $35-57 \%$ in different East Asian subpopulations, results in markedly reduced alcohol tolerance $[3,4]$. People carrying the mutant $A L D H 2$ allele display characteristic acetaldehyde-mediated acute effects of alcohol drinking such as facial flushing and increased pulse rate [21]. The mutation is caused by a single nucleotide substitution of $\mathrm{G}$ for $\mathrm{A}$ in exon 12 , resulting in the replacement of glutamate to lysine at position 504 of the protein [3]. Glu504 is located within the oligomerization domain at the dimer interface of the tetrameric enzyme which is critical for the formation of both dimer and tetramer [22]. In wildtype ALDH2 (ALDH2*1), Glu504 forms hydrogen bonds with Arg281 of the same subunit and with Arg492 of the adjacent dimer partner. The disruption of these interactions by the presence of Lys504 in the mutant (ALDH2*2) perturbs the structure of the subunit with the mutation as well as its dimer partner [23]. Binding of the coenzyme $\mathrm{NAD}^{+}$ to $\mathrm{ALDH}_{2}^{*} 2$ is impaired, and the mutant enzyme has an increased $K_{m}$ for $\mathrm{NAD}^{+}$and a decreased $k_{\text {cat }}$, which leads to a very low enzymatic activity in vivo [24]. Therefore, ALDH2*2 acts in a dominant-negative manner. The phenotypic loss of $\mathrm{ALDH} 2$ activity is found in both heterozygous $\left(A L D H 2^{*} 1{ }^{*} 2\right)$ and homozygous $\left(A L D H^{*} 2 /^{*} 2\right)$ individuals, whose blood acetaldehyde concentrations are approximately 6 and 19 times higher than those with active ALDH2, respectively, following a low to moderate intake of alcohol [25].

\section{Glu504Lys SNP of $A L D H 2$ Gene and the Risk of Human Diseases}

The high blood acetaldehyde levels in individuals with $A L D H 2^{*} 2$ allele after moderate alcohol consumption mediate the enhanced alcohol sensitivity in this population [26]. In comparison to $A L D H 2^{*} 1 /{ }^{*} 1$ homozygotes, individuals with $A L D H 2^{*} 1 /^{*} 2$ genotype experience significantly higher pulse rate and greater facial flushing as early as $30 \mathrm{~min}$ following alcohol consumption and have higher risk of developing hangover symptoms [21, 27]. Electroencephalographs (ECG) show that the increases in P300 latency and decreases in P300 amplitude following alcohol consumption are greater in individuals with $A L D H 2^{*} 1 /{ }^{*} 2$ genotype than in individuals with $A L D H 2^{*} 1{ }^{*} 1$ genotype, suggesting that their cognitive functioning may be more impaired by alcohol exposure [28, 29]. Individuals with $A L D H 2^{*} 1 /^{*} 2$ genotype also show more impaired psychomotor performance including reaction time to complex visual information, visuomotor coordination, and exact motor ability than those with $A L D H 2^{*} 1 /^{*} 1$ at 30 and 60 min after moderate alcohol consumption $[29,30]$. Because of these acute effects after alcohol ingestion, Glu504Lys SNP of $A L D H 2$ gene is protective against the development of alcoholism and perhaps may decrease the risk of chronic diseases caused by alcohol overconsumption. On the other hand, individuals carrying Glu504Lys SNP who do drink alcohol may have an increased incidence of alcohol-mediated diseases. Furthermore, for susceptible individuals that drink limited amount of alcohol, defective ALDH2 may cause the accumulation of toxic aldehydes, which can be generated endogenously from metabolism other than ethanol oxidation or directly enter the body from foods and the environment, leading to enhanced oxidative stress [31] and impaired cell function and subsequently affecting the risk of a variety of human chronic diseases.

4.1. CVD and Associated Diseases. Although liver is considered as the primary site for ethanol oxidation, other organs such as heart also participate in ethanol metabolism. Elevated acetaldehyde can lead to cardiac toxicity and plays a significant role in the pathogenesis of alcoholic cardiomyopathy (reviewed in [32]). Overexpression of the ALDH2 gene alleviates oxidative stress and apoptosis induced by ethanol and acetaldehyde in human cardiac myocytes [33] and mitigates mechanical anomalies induced by alcohol in myocardium of ALDH2 transgenic mice [34], suggesting that the detoxification of acetaldehyde by ALDH2 protects against alcohol-induced cardiac toxicity. In addition, accumulation of cytotoxic aldehydes other than acetaldehyde, which are either generated during reactive oxygen species (ROS)-induced stress or ingested from foods or polluted environment, such as 4 -HNE and acrolein, contributes to the oxidative stress and impairs cardiac functions [35-37]. 4HNE can be endogenously produced from lipid peroxidation of polyunsaturated fatty acids in conditions like ischemia and reperfusion of heart. In fact, accumulation of 4-HNE-protein adducts and protein carbonyls has been detected in the failing hearts [38] and has been shown to depress contractility of 
isolated cardiac myocytes, inhibit mitochondrial function, and cause tissue damage after cardiac ischemia [39, 40]. Another toxic aldehyde acrolein is present in a variety of foods and is detected in high levels in cigarette smoke and overheated oils (reviewed in [18]). In a mouse model of acute myocardial infarction, dietary treatment with acrolein at concentration $(5 \mathrm{mg} / \mathrm{kg})$ comparable to human diet, $24 \mathrm{~h}$ prior to a $30 \mathrm{~min}$ coronary artery occlusion and $24 \mathrm{~h}$ reperfusion, significantly increases myocardial infarct size, exacerbating cardiac injury caused by ischemia and reperfusion [41]. Acrolein also blocks cardioprotective effects induced by the pretreatment of $\mathrm{NO}$ donor diethylenetriamine/NO via mechanisms that disrupt protein kinase $\mathrm{C} \varepsilon$ signal transduction [41]. Thus, detoxification of toxic acetaldehydes such as those mentioned above by ALDH2 could be beneficial for prevention and intervention of CVD. In a rat model where heart failure is induced by a 6-week treatment of myocardial infarction, treatment of a selective ALDH2 activator Alda1, starting 4 weeks after myocardial infarction, significantly decreases the accumulation of 4-HNE and its associated cell toxicity in failing hearts and improves cardiomyocyte shortening, left ventricular compliance, and diastolic function [38]. Consistently, overexpression of $A L D H 2$ gene in mice decreases 4-HNE levels elevated by ischemia and reperfusion and significantly alleviates ischemia/reperfusion injury and hypoxia/reoxygenation-induced cardiomyocyte contractile dysfunction. In contrast, the accumulation of cardiac 4-HNE and the cardiac injury in response to ischemia-reperfusion are exacerbated in $A L D H 2$ knockout mice [42].

These findings suggest that disruption of ALDH2 activity may increase the susceptibility of an individual to CVD. Recently, two meta-analyses have shown that Glu504Lys SNP of $A L D H 2$ gene in Asian populations is associated with increased risk of coronary artery disease (odds ratio $(\mathrm{OR})=$ 1.36 and $1.28,95 \%$ confidence interval $(\mathrm{CI})=1.06-1.75$ and $1.10-1.48$, and $p=0.017$ and 0.001 , resp.) and myocardial infarction $(\mathrm{OR}=1.64$ and $1.58,95 \% \mathrm{CI}=1.22-2.20$ and $1.15-$ 2.19 , and $p=0.001$ and 0.005 , resp.) $[6,7]$. It has been reported that $A L D H 2^{*} 2$ allele is associated with low serum HDL cholesterol levels in Asian populations [43]. In addition, as ALDH2 also functions in the formation of nitric oxide from nitroglycerin, Glu504Lys SNP of ALDH2 gene eliminates the activity of the enzyme to catalyze the reaction. Therefore, it is not surprising that $A L D H 2^{*} 2$ allele is associated with a lack of an efficacious clinical response to nitroglycerin treatment for coronary heart disease [44].

Surprisingly, $A L D H 2^{*} 2$ carriers with chronic cyanosis are shown to have unexpectedly greater tolerance to ischaemia and reperfusion injury in comparison to $A L D H 2^{*} 1$ homozygotes. $A L D H 2^{*} 2$ carriers have lower postoperative troponin I levels and inotropic scores as well as shorter length of intensive care unit (ICU) and hospital stay after open-heart surgery [45]. It has been found that aldehyde accumulation caused by cyanosis in $A L D H 2^{*} 2$ carriers results in larger myocardium glutathione (GSH) pools [45]. The increased intracellular GSH levels are also seen in the hearts of $A L D H 2^{*} 2$ transgenic mice when compared with those of wild-type controls [46]. This compensatory myocardium GSH pool may contribute to the unexpectedly better cardioprotection seen in the $A L D H 2^{*} 2$ patients [45].

4.2. Hypertension. Hypertension is a major risk factor for CVD. It is known that excessive alcohol consumption promotes the development of hypertension. The effects of Glu504Lys SNP of ALDH2 gene on blood pressure are complicated by alcohol consumption and the presence of other genetic polymorphisms. It has been found that the prevalence of hypertension is higher in $A L D H 2^{*} 1$ homozygotes ( $\mathrm{OR}=$ $1.67,95 \% \mathrm{CI}=1.37-2.08$, and $p<0.0001$ ) in comparison to $A L D H 2^{*} 2$ carriers among males in a Japanese population. Further investigation on $A L D H 2$ genotypes and the level of alcohol consumption suggests that the $A L D H 2^{*} 1 /^{*} 1$ genotype correlates with increased risk for hypertension among males primarily through its association with the level of alcohol consumption [47]. Similar results have been reported by Amamoto et al. The authors have found that $A L D H 2^{*} 2$ carriers have lower incidence of hypertension than $A L D H 2^{*} 2$ noncarriers $(\mathrm{OR}=0.67,95 \% \mathrm{CI}=0.47-0.96, p=0.030)$, while this correlation is not observed in individuals whose alcohol consumption is below median level or in the group not taking antihypertensive agents [48]. A case-control study later has shown that $A L D H 2^{*} 1{ }^{*} 1$ genotype is an independent risk factor for essential hypertension among males. The OR for the presence of hypertension for $A L D H 2^{*} 1 /^{*} 1$ genotype compared with other genotypes is $1.93(95 \% \mathrm{CI}=1.12-$ $3.31, p=0.018$ ) [49]. In Japanese male regular drinkers $(\geq 22 \mathrm{~g}$ ethanol/d), Tsuchihashi-Makaya et al. have reported that $A L D H 2^{*} 1 /^{*} 1$ genotype is an independent predictor for increased systolic $(\beta$-coefficient $=2.96, p=0.03)$ and diastolic $(\beta$-coefficient $=2.26, p=0.01)$ blood pressure after adjusting for alcohol consumption [50]. More recently, it has also been observed in a Chinese Han population that $A L D H 2^{*} 2$ carriers who drink alcohol have lower risk of essential hypertension $(\mathrm{OR}=0.55,95 \% \mathrm{CI}=0.36-0.85)$, while this association is not found in nondrinkers [51]. It is proposed that the vasodilating effect of acetaldehyde [52] may be one of the mechanisms for the lower blood pressure seen in the $A L D H 2^{*} 2$ carriers who drink alcohol [50].

Conversely, Chang et al. have reported that $A L D H 2^{*} 2$ allele is significantly associated with increases of blood pressure (systolic blood pressure: $0.865 \mathrm{mmHg} / \mathrm{yr}$, diastolic blood pressure: $0.537 \mathrm{mmHg} / \mathrm{yr}$ ) in a prospective Chinese cohort followed on an average of 5.7 yrs [53]. The authors suggest that carriers with $A L D H 2^{*} 2$ allele may be more susceptible to progress to hypertension compared with noncarriers [53]. The size and characteristics (age, gender distribution, eating habits, average amount of alcohol consumption, medications, etc.) of the population examined may cause the inconsistent results seen in different studies. A total of 753 individuals from 276 families are included in the follow-up study mentioned above [53]; it is possible that other genetic polymorphisms may exist in this population and act together with $A L D H 2$ genotype in combination with dietary/environmental factors to promote hypertension. Indeed, the benefit of $A L D H 2^{*} 2$ allele on blood pressure has been shown to disappear when the effect is evaluated in 
combination with SOD2 polymorphism. Individuals carrying both $A L D H 2^{*} 2$ allele and SOD2 $\mathrm{Val} / \mathrm{Val}$ genotype have a significantly higher risk of hypertension among drinkers than in nondrinkers (adjusted OR $=6.22$, 95\% CI $=2.26-$ 17.1, and $p<0.001$ ) [54]. Therefore, both lifestyle factors such as alcohol drinking and other genetic variations may have impacts on the susceptibility of $A L D H 2$ genotypes to hypertension.

4.3. Cancer. Aldehydes are very reactive molecules, which can modify proteins and nucleic acids, causing dysfunction of these biomolecules. Binding of acetaldehyde with DNA has been demonstrated to promote carcinogenesis in laboratory animals and alcoholic individuals $[55,56]$. Indeed, chronic alcohol consumption has been shown to be a strong risk factor for the cancer of many tissues and organs including the upper aerodigestive tract (oral cavity, pharynx, larynx, and oesophagus), liver, colorectum, and breast [57, 58]. Acetaldehyde is considered to be one of the important mechanisms contributing to the development of alcoholassociated cancers $[55,56]$.

The effect of the alteration of ALDH2 activity by Glu504Lys SNP on the risk of cancer has been shown to interact with lifestyle factors, especially alcohol consumption. Yokoyama et al. have reported that, in male Japanese alcoholics, the $A L D H 2 * 2$ allele significantly increases the risks (OR) for the oro-pharyngo-laryngeal (11.14), esophageal (12.50), stomach (3.49), colon (3.35), and lung (8.20) cancer, but not for liver or other cancers after adjustment for age, daily alcohol consumption, and amount of cigarette smoking [8]. Further evaluation of the association of ALDH2 Glu504Lys SNP with esophageal cancer has demonstrated that $\mathrm{OR}$ for the $A L D H 2^{*} 1 /^{*} 2$ and $A L D H 2^{*} 2 /^{*} 2$ genotypes in comparison to the $A L D H 2^{*} 1 /^{*} 1$ genotype is $3.43(95 \%$ $\mathrm{CI}=1.74-6.75)$ after adjustment for age, sex, drinking, and smoking status. A strong gene-environment interaction exists between $A L D H 2^{*} 2$ allele and excessive alcohol consumption for the risk of esophageal cancer. The OR for heavy drinkers with $A L D H 2^{*} 2$ relative to nonheavy drinkers with ALDH $2^{*} 1 /^{*} 1$ genotype is 6.84 (95\% CI = 2.39-19.6) [59]. A case-control study on patients with esophageal squamous cell carcinoma from Taiwan has shown similar results. In the study, it has been found that individuals with $A L D H 2^{*} 1{ }^{*} 2$ and $A L D H 2^{*} 2 /^{*} 2$ genotypes have 4.99- (95\% CI = 3.11-7.99) and 4.24 -fold (95\% CI $=1.52-11.84)$ risk, respectively, of developing esophageal cancer, when compared with those with $A L D H 2^{*} 1 /^{*} 1$ genotype, after adjustment for appropriate covariates. And the heavy drinkers $(\geq 1,200 \mathrm{~g} /$ year $)$ with $A L D H 2^{*} 1 /^{*} 2$ genotype have 30.53 -fold risk (95\% CI $=12.01-$ 77.64) of developing esophageal cancer in comparison to nondrinkers with $A L D H 2^{*} 1 /^{*} 1[60]$.

ALDH2 Glu504Lys polymorphism has also been shown to interact with the SNPs of other key enzymes in ethanol metabolism in the development of alcohol-associated cancers [61]. Alcohol dehydrogenase $1 \mathrm{~B}(\mathrm{ADH} 1 \mathrm{~B})$ is one of the major enzymes belonging to a group of $\mathrm{ADH}$ that break down ethanol to acetaldehyde. Arg47His SNP of $A D H 1 B\left(A D H 1 B^{*} 2\right)$, which exists in more than $90 \%$ of
East Asians, encodes a superactive subunit of ADH1B that promotes the accumulation of acetaldehyde after alcohol drinking [61]. In Japanese alcoholic patients, blood levels of N(2)-ethylidene- $2^{\prime}$-deoxyguanosine (N(2)-ethylidene$\mathrm{dG}$ ), the most abundant acetaldehyde-derived DNA adduct, are remarkably higher in individuals carrying both $A L D H 2^{*} 2$ and $A D H 1 B^{*} 2$ alleles, suggesting that alcoholic individuals with both SNPs may accumulate more DNA damage and may have increased susceptibility to cancer development [62]. Surprisingly, a study on Japanese alcoholic men (age > 40 y) has shown that individuals with $A D H 1 B^{*} 1 /{ }^{*} 1$ genotype $(\mathrm{OR}=2.03)$ have increased risk of esophageal cancer after adjustment for drinking and smoking. $A L D H 2^{*} 1 /^{*} 2$ genotype also had higher risk of esophageal cancer $(\mathrm{OR}=12.76)$. For individuals with $A L D H 2^{*} 1 /^{*} 2$ and $A D H 1 B^{*} 1 /^{*} 1$ genotypes, the esophageal cancer risk is enhanced in a multiplicative fashion $(\mathrm{OR}=27.66)$ [63]. A few mechanisms are proposed for this unexpected result. First, it has been demonstrated that the lower systemic elimination of ethanol from the body by $A D H 1 B^{*} 1{ }^{*} 1$ may lead to increased production of acetaldehyde by oral microbes, thus prolonging the exposure to acetaldehyde through saliva [64]. In addition, individuals who carry the highly active $A D H 1 B^{*} 2$ allele rapidly convert ethanol to acetaldehyde following alcohol consumption, leading to the accumulation of acetaldehyde and the facial flushing syndrome. The unpleasant symptoms prevent $A D H 1 B^{*} 2$ carriers from drinking alcohol, therefore perhaps exerting a protective effect against alcohol-associated cancer development [57].

Recently, studies have confirmed that the $A L D H 2^{*} 2$ allele is associated with an increased risk of gastric cancer [65, 66]. It has also been found that there is an interaction between $A L D H 2$ Glu504Lys SNP and alcohol consumption in the development of gastric cancer [65]. The studies on the association of the ALDH2 Glu504Lys SNP with the risk of colorectal cancer have given inconsistent results. Two recent meta-analyses indicate that $A L D H 2$ Glu504Lys SNP may be associated with a decreased risk of colorectal cancer $[67,68]$. No significant impact of Glu504Lys SNP of ALDH2 gene on the risk of hepatocellular carcinoma [69] and breast cancer has been found in East Asian populations [70, 71].

4.4. Alzheimer's Disease. $\mathrm{AD}$ is the most common neurodegenerative disease that causes dementia in the elderly. The major pathological characteristics of $\mathrm{AD}$ brains are the presence of senile plaques composed of beta-amyloid peptide (Abeta), neurofibrillary tangles (NFT) formed by hyperphosphorylated tau protein, and neuronal loss (reviewed in $[72,73])$. Accumulating evidence has shown that oxidative stress is one of the important factors in the pathogenesis of $\mathrm{AD}$ (reviewed in [74]). Products of lipid peroxidation such as 4-HNE have been reported to be elevated in the brains of $\mathrm{AD}$ patients [75]. It has been found that 4-HNE can induce neuronal death and synapse dysfunction [76] and markedly inhibit microtubule formation and neurite outgrowth [77]. 4-HNE has also been shown to react with phosphorylated tau and induce conformational changes in tau proteins that promote the formation of NFT $[78,79]$. 
In addition, exposure of NT(2) neurons to 4-HNE elicits an upregulation of the expression of beta-site amyloid precursor protein cleaving enzyme (BACE), causing significant increase in intracellular and secreted levels of Abeta peptides [80]. Thus, elevated levels of 4-HNE in central nervous system may contribute to the pathogenesis of $\mathrm{AD}$. In PC12 cells, ALDH2 deficiency produced by introducing $A L D H 2^{*} 2$ gene leads to marked accumulation of 4-HNE in response to oxidative stress stimuli and increased vulnerability to 4-HNE-induced cell death [81]. Similarly, central neurons from transgenic mice overexpressing $A L D H 2^{*} 2$ gene are more sensitive to 4-HNE-induced toxicity than cells from control animals [82]. Moreover, these ALDH2 deficient mice exhibit an agedependent decrease in spatial cognitive ability starting at the age of 6 months [82]. The reduced resistance to oxidative stress has been proposed as one mechanism that leads to the neurodegeneration and memory loss in ALDH2 deficient mice [82]. These results suggest that ALDH2 Glu504Lys SNP could be a genetic risk factor for AD in susceptible populations.

A case-control study from Japan has shown that the number of individuals carrying $A L D H 2^{*} 2$ allele is significantly higher in the patients with late-onset $\mathrm{AD}$ (LOAD) than in the controls $(48.1 \%$ versus $37.4 \%, p=0.001)$ [5]. $A L D H 2^{*} 2$ genotype interacts synergistically with the presence of the apolipoprotein E allele 4 (APOE- 4 ), which is a widely accepted risk factor for LOAD [5]. Logistic regression analysis shows that $A L D H 2^{*} 2$ allele increases the risk for LOAD independently of APOE- $\varepsilon 4(p=0.002)$ status, while the coexistence of the APOE- 84 allele and $A L D H 22^{*} 2$ allele synergistically increases the frequency of LOAD, which is 31 times higher in individuals being APOE- $\varepsilon 4$ homozygous and having at least one $A L D H 2^{*} 2$ allele than in those having neither allele [5]. Moreover, in LOAD patients homozygous for $A P O E-\varepsilon 4$, the age at onset of LOAD is significantly younger in those with $A L D H 2^{*} 2$ allele than in those without $A L D H 2^{*} 2$ allele, and the dosage of the $A L D H 2^{*} 2$ allele significantly affects the age at onset of the disease [5]. Interestingly, immunostaining of the brain of $\mathrm{AD}$ patients using anti-4-HNE antibody reveals that the cytoplasm of pyramidal cell is positive for 4-HNE only in individuals with $A P O E-\varepsilon 4$ allele [83]. Studies have shown that APOE proteins interact with 4-HNE and the strength of binding between APOE isoforms and 4-HNE is different, with the order $\varepsilon 2$ $>\varepsilon 3>\varepsilon 4$. This correlates with the differential protective effect of APOE isoforms against 4-HNE-induced neuronal apoptosis [84]. These results suggest that APOE may have an important role in elimination of 4-HNE. And the possession of APOE- $\varepsilon 4$, the APOE with the weakest 4-HNE binding ability, may lead to the accumulation of toxic 4-HNE in neurons, which could be further intensified by the reduction of ALDH2 activity, resulting in increased oxidative stress and higher risk for developing $\mathrm{AD}$ [82].

It has been observed in a Chinese case-control study that individuals carrying $A L D H 2^{*} 2$ allele have significantly higher risk of $\mathrm{AD}(\mathrm{OR}=3.11,95 \% \mathrm{CI}=2.06-4.69$, and $p<$ 0.001 ) [85]. In contrast, a study on sporadic $\mathrm{AD}$ patients from Mongolic ethnic group in China has not found an association between $A L D H 2^{*} 2$ allele and the risk of $\mathrm{AD}$ [86]. There may be several reasons underlying the discrepancies found in these studies. First, the populations analyzed are from different ethnic backgrounds or different geographic regions; thus it is not impossible that other genetic polymorphisms may have an impact on the results. Secondly, environmental factors such as eating habits or alcohol consumption of the studied subjects should be considered and may influence the results. In addition, the sample size as well as the distribution of male/female subjects in the studies may affect the significance of the result. Recently, a meta-analysis evaluated the association of $A L D H 2$ variants with the risk of $\mathrm{AD}$ in East Asian populations has found that $A L D H 2{ }^{*} 1 /{ }^{*} 2$ and ${ }^{*} 2 /^{*} 2$ genotypes are associated with increased AD risk only in subgroup analyses in which male subjects are included (OR $=1.72,95 \% \mathrm{CI}=1.10-2.67$, and $p=0.02)$ [87].

\section{Conclusions}

ALDH2 is the major enzyme for the clearance of ethanol metabolite acetaldehyde. It is also important for our body to metabolize other toxic aldehydes, such as lipid peroxidation product 4-HNE, generated endogenously or ingested from environment. Dysfunction of ALDH2 in individuals carrying $A L D H 2$ Glu504Lys SNP leads to increased accumulation of toxic aldehydes that may result in higher risk of a variety of human diseases including CVD, cancer, and AD. Here we discuss the evidence that implicates $A L D H 2^{*} 2$ allele as a candidate genetic risk factor for these chronic diseases. It has to be noted that the pathogenesis of these chronic diseases involves multiple mechanisms; thus the interactions between $A L D H 2^{*} 2$ allele and other genetic polymorphisms as well as the gene-environment interactions have to be considered to give a more thorough picture on how $A L D H 2$ genotype affects the development of chronic diseases. In particular, the Glu504Lys SNP of $A L D H 2$ gene is closely related to alcohol drinking behavior. For alcoholics, $A L D H 2^{*} 2$ allele may significantly exacerbate the risk of alcohol-related health problems. On the other hand, the unpleasant flushing syndromes caused by the accumulation of acetaldehyde result in less alcohol consumption and reduced incidence of alcoholism in individuals carrying $A L D H 2^{*} 2$ allele. As a consequence, for pathogenesis directly associated with ethanol consumption, Glu504Lys SNP of $A L D H 2$ gene can be a protective factor for $A L D H 2^{*} 2$ allele carriers who limit their exposure to ethanol. In addition, the effect of Glu504Lys SNP of ALDH2 gene on the development of chronic diseases, which are often multifactorial, can be complicated by other genetic variations [54]. In summary, defective ALDH2 activity may compromise the elimination of reactive aldehydes, leading to increased cytotoxicity and oxidative stress. Other genetic or environmental/life style factors, which promote (or inhibit) the stress caused by defective ALDH2, may increase (or reduce) the susceptibility of individuals carrying $A L D H 2^{*} 2$ allele to relevant chronic diseases (Figure 1).

More effort should be spent in the future to understand the biochemical and molecular mechanisms underlying the association of $A L D H 2$ Glu504Lys SNP with chronic diseases. Studies using animal and cell models with defective ALDH2 


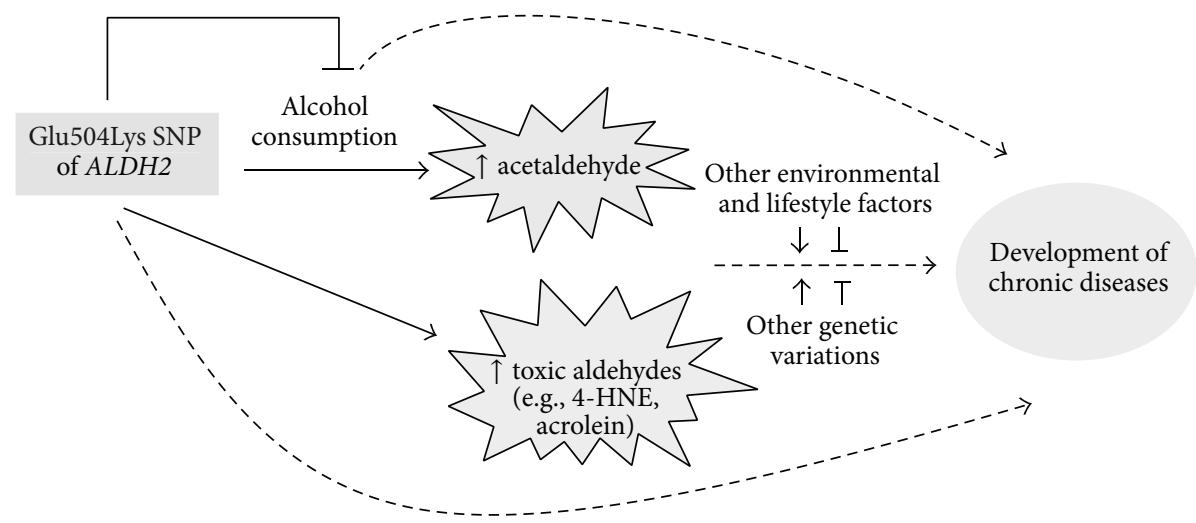

FIGURE 1: The association of Glu504Lys SNP of $A L D H 2$ gene with human diseases is complicated by other genetic variations and environmental/lifestyle factors in addition to alcohol drinking.

activity will further our knowledge on the molecular basis of human phenotype of $A L D H 2$ variant and provide information for discovery of potential interventions and therapeutics targeting ALDH2.

Finally, for those diseases that are prompted synergistically by ALDH2 Glu504Lys SNP and alcohol consumption, individuals carrying $A L D H 2^{*} 2$ allele should be targeted for reducing alcohol exposure as part of the preventive strategy.

\section{Conflict of Interests}

The authors declare that there is no conflict of interests regarding the publication of this paper.

\section{Acknowledgments}

This work is supported by grants from the National Natural Science Foundation of China (31371082), research funds from Harbin Institute of Technology at Weihai (HIT(WH)Y200902 and HIT(WH)Y200903), and Weihai Science and Technology Development Program (2011DXGJ14).

\section{References}

[1] T. Isse, K. Matsuno, T. Oyama, K. Kitagawa, and T. Kawamoto, "Aldehyde dehydrogenase 2 gene targeting mouse lacking enzyme activity shows high acetaldehyde level in blood, brain, and liver after ethanol gavages," Alcoholism: Clinical and Experimental Research, vol. 29, no. 11, pp. 1959-1964, 2005.

[2] A. A. Klyosov, L. G. Rashkovetsky, M. K. Tahir, and W.M. Keung, "Possible role of liver cytosolic and mitochondrial aldehyde dehydrogenases in acetaldehyde metabolism," Biochemistry, vol. 35, no. 14, pp. 4445-4456, 1996.

[3] D. W. Crabb, H. J. Edenberg, W. F. Bosron, and T.-K. Li, "Genotypes for aldehyde dehydrogenase deficiency and alcohol sensitivity. The inactive ALDH2(2) allele is dominant," The Journal of Clinical Investigation, vol. 83, no. 1, pp. 314-316, 1989.

[4] H. E. Goedde, D. P. Agarwal, S. Harada et al., "Population genetic studies on aldehyde dehydrogenase isozyme deficiency and alcohol sensitivity," The American Journal of Human Genetics, vol. 35, no. 4, pp. 769-772, 1983.
[5] K. Kamino, K. Nagasaka, M. Imagawa et al., "Deficiency in mitochondrial aldehyde dehydrogenase increases the risk for late-onset Alzheimer's disease in the Japanese population," Biochemical and Biophysical Research Communications, vol. 273, no. 1, pp. 192-196, 2000.

[6] Q. Wang, S. Zhou, L. Wang et al., “ $A L D H 2$ rs671 polymorphism and coronary heart disease risk among asian populations: a meta-analysis and meta-regression," DNA and Cell Biology, vol. 32, no. 7, pp. 393-399, 2013.

[7] J.-Y. Gu and L.-W. Li, "ALDH2 Glu504Lys polymorphism and susceptibility to coronary artery disease and myocardial infarction in East Asians: a meta-analysis," Archives of Medical Research, vol. 45, no. 1, pp. 76-83, 2014.

[8] A. Yokoyama, T. Muramatsu, T. Ohmori et al., "Alcohol-related cancers and aldehyde dehydrogenase-2 in Japanese alcoholics," Carcinogenesis, vol. 19, no. 8, pp. 1383-1387, 1998.

[9] V. Vasiliou and D. W. Nebert, "Analysis and update of the human aldehyde dehydrogenase (ALDH) gene family," Human Genomics, vol. 2, no. 2, pp. 138-143, 2005.

[10] S. Futterman, "Enzymatic oxidation of vitamin A aldehyde to vitamin A acid," The Journal of Biological Chemistry, vol. 237, pp. 677-680, 1962.

[11] M.-K. Chern and R. Pietruszko, "Human aldehyde dehydrogenase E3 isozyme is a betaine aldehyde dehydrogenase," Biochemical and Biophysical Research Communications, vol. 213, no. 2, pp. 561-568, 1995.

[12] A. Kikonyogo and R. Pietruszko, "Aldehyde dehydrogenase from adult human brain that dehydrogenates $\gamma$-aminobutyraldehyde: purification, characterization, cloning and distribution," Biochemical Journal, vol. 316, no. 1, pp. 317-324, 1996.

[13] N. Seiler and B. Eichentopf, "4 Aminobutyrate in mammalian putrescine catabolism," Biochemical Journal, vol. 152, no. 2, pp. 201-210, 1975.

[14] J. W. Fisher, D. Mahle, and R. Abbas, "A human physiologically based pharmacokinetic model for trichloroethylene and its metabolites, trichloroacetic acid and free trichloroethanol," Toxicology and Applied Pharmacology, vol. 152, no. 2, pp. 339359, 1998.

[15] M. M. Anderson, S. L. Hazen, F. F. Hsu, and J. W. Heinecke, "Human neutrophils employ the myeloperoxidase-hydrogen peroxide-chloride system to convert hydroxy-amino acids into glycolaldehyde, 2-hydroxypropanal, and acrolein: a mechanism 
for the generation of highly reactive $\alpha$-hydroxy and $\alpha, \beta$ unsaturated aldehydes by phagocytes at sites of inflammation," The Journal of Clinical Investigation, vol. 99, no. 3, pp. 424-432, 1997.

[16] V. J. Feron, H. P. Til, F. de Vrijer, R. A. Woutersen, F. R. Cassee, and P. J. van Bladeren, "Aldehydes: occurrence, carcinogenic potential, mechanism of action and risk assessment," Mutation Research/Genetic Toxicology, vol. 259, no. 3-4, pp. 363-385, 1991.

[17] S. Srivastava, A. Chandra, L.-F. Wang et al., "Metabolism of the lipid peroxidation product, 4-hydroxy-trans-2-nonenal, in isolated perfused rat heart," The Journal of Biological Chemistry, vol. 273, no. 18, pp. 10893-10900, 1998.

[18] D. P. Ghilarducci and R. S. Tjeerdema, "Fate and effects of acrolein," Reviews of Environmental Contamination and Toxicology, vol. 144, pp. 95-146, 1995.

[19] K. Sydow, A. Daiber, M. Oelze et al., "Central role of mitochondrial aldehyde dehydrogenase and reactive oxygen species in nitroglycerin tolerance and cross-tolerance," The Journal of Clinical Investigation, vol. 113, no. 3, pp. 482-489, 2004.

[20] M. J. Stewart, K. Malek, and D. W. Crabb, "Distribution of messenger RNAs for aldehyde dehydrogenase 1, aldehyde dehydrogenase 2, and aldehyde dehydrogenase 5 in human tissues," Journal of Investigative Medicine, vol. 44, no. 2, pp. 42-46, 1996.

[21] K.-Y. Bae, S.-W. Kim, H.-Y. Shin et al., "The acute effects of ethanol and acetaldehyde on physiological responses after ethanol ingestion in young healthy men with different ALDH2 genotypes," Clinical Toxicology, vol. 50, no. 4, pp. 242-249, 2012.

[22] C. G. Steinmetz, P. Xie, H. Weiner, and T. D. Hurley, "Structure of mitochondrial aldehyde dehydrogenase: the genetic component of ethanol aversion," Structure, vol. 5, no. 5, pp. 701-711, 1997.

[23] J. Farrés, X. Wang, K. Takahashi, S. J. Cunningham, T. T. Wang, and $\mathrm{H}$. Weiner, "Effects of changing glutamate 487 to lysine in rat and human liver mitochondrial aldehyde dehydrogenase: a model to study human (oriental type) class 2 aldehyde dehydrogenase," The Journal of Biological Chemistry, vol. 269, no. 19, pp. 13854-13860, 1994.

[24] H. N. Larson, H. Weiner, and T. D. Hurley, "Disruption of the coenzyme binding site and dimer interface revealed in the crystal structure of mitochondrial aldehyde dehydrogenase 'Asian' variant,' Journal of Biological Chemistry, vol. 280, no. 34, pp. 30550-30556, 2005.

[25] G.-S. Peng and S.-J. Yin, "Effect of the allelic variants of aldehyde dehydrogenase ALDH2 2 and alcohol dehydrogenase $\mathrm{ADH}_{1}{ }^{*} 2$ on blood acetaldehyde concentrations," Human Genomics, vol. 3, no. 2, pp. 121-127, 2009.

[26] T. L. Wall, C. M. Peterson, K. P. Peterson et al., "Alcohol metabolism in Asian-American men with genetic polymorphisms of aldehyde dehydrogenase," Annals of Internal Medicine, vol. 127, no. 5, pp. 376-379, 1997.

[27] M. Yokoyama, A. Yokoyama, T. Yokoyama et al., "Hangover susceptibility in relation to aldehyde dehydrogenase-2 genotype, alcohol flushing, and mean corpuscular volume in Japanese workers," Alcoholism: Clinical and Experimental Research, vol. 29, no. 7, pp. 1165-1171, 2005.

[28] T. L. Wall and C. L. Ehlers, "Acute effects of alcohol on P300 in Asians with different ALDH2 genotypes," Alcoholism: Clinical and Experimental Research, vol. 19, no. 3, pp. 617-622, 1995.

[29] H.-Y. Shin, I.-S. Shin, and J.-S. Yoon, "ALDH2 genotypeassociated differences in the acute effects of alcohol on P300, psychomotor performance, and subjective response in healthy young Korean men: a double-blind placebo-controlled crossover study," Human Psychopharmacology, vol. 21, no. 3, pp. 159-166, 2006.

[30] S.-W. Kim, K.-Y. Bae, H.-Y. Shin et al., “The role of acetaldehyde in human psychomotor function: a double-blind placebocontrolled crossover study," Biological Psychiatry, vol. 67, no. 9, pp. 840-845, 2010.

[31] I. Ohsawa, K. Kamino, K. Nagasaka et al., "Genetic deficiency of a mitochondrial aldehyde dehydrogenase increases serum lipid peroxides in community-dwelling females," Journal of Human Genetics, vol. 48, no. 8, pp. 404-409, 2003.

[32] Y. Zhang and J. Ren, "ALDH2 in alcoholic heart diseases: molecular mechanism and clinical implications," Pharmacology and Therapeutics, vol. 132, no. 1, pp. 86-95, 2011.

[33] S.-Y. Li, Q. Li, J. J. Shen et al., "Attenuation of acetaldehydeinduced cell injury by overexpression of aldehyde dehydrogenase-2 (ALDH2) transgene in human cardiac myocytes: role of MAP kinase signaling," Journal of Molecular and Cellular Cardiology, vol. 40, no. 2, pp. 283-294, 2006.

[34] T. A. Doser, S. Turdi, D. P. Thomas, P. N. Epstein, S.-Y. Li, and J. Ren, "Transgenic overexpression of aldehyde dehydrogenase2 rescues chronic alcohol intake-induced myocardial hypertrophy and contractile dysfunction," Circulation, vol. 119, no. 14, pp. 1941-1949, 2009.

[35] S. Mak, D. C. Lehotay, M. Yazdanpanah, E. R. Azevedo, P. P. Liu, and G. E. Newton, "Unsaturated aldehydes including 4-OHnonenal are elevated in patients with congestive heart failure," Journal of Cardiac Failure, vol. 6, no. 2, pp. 108-114, 2000.

[36] P. Kaplan, Z. Tatarkova, P. Racay, J. Lehotsky, M. Pavlikova, and D. Dobrota, "Oxidative modifications of cardiac mitochondria and inhibition of cytochrome $c$ oxidase activity by 4hydroxynonenal," Redox Report, vol. 12, no. 5, pp. 211-218, 2007.

[37] M. A. Ismahil, T. Hamid, P. Haberzettl et al., "Chronic oral exposure to the aldehyde pollutant acrolein induces dilated cardiomyopathy," The American Journal of Physiology-Heart and Circulatory Physiology, vol. 301, no. 5, pp. H2050-H2060, 2011.

[38] K. M. S. Gomes, J. C. Campos, L. R. G. Bechara et al., "Aldehyde dehydrogenase 2 activation in heart failure restores mitochondrial function and improves ventricular function and remodelling," Cardiovascular Research, vol. 103, no. 4, pp. 498508, 2014.

[39] N. S. Aberle II, M. J. Picklo Sr., V. Amarnath, and J. Ren, "Inhibition of cardiac myocyte contraction by 4-hydroxy-trans2-nonenal," Cardiovascular Toxicology, vol. 4, no. 1, pp. 21-28, 2004.

[40] D. T. Lucas and L. I. Szweda, "Cardiac reperfusion injury: aging, lipid peroxidation, and mitochondrial dysfunction," Proceedings of the National Academy of Sciences of the United States of America, vol. 95, no. 2, pp. 510-514, 1998.

[41] G.-W. Wang, Y. Guo, T. M. Vondriska et al., "Acrolein consumption exacerbates myocardial ischemic injury and blocks nitric oxide-induced PKC $\varepsilon$ signaling and cardioprotection," Journal of Molecular and Cellular Cardiology, vol. 44, no. 6, pp. 1016-1022, 2008.

[42] H. Ma, R. Guo, L. Yu, Y. Zhang, and J. Ren, "Aldehyde dehydrogenase 2 (ALDH2) rescues myocardial ischaemia/reperfusion injury: role of autophagy paradox and toxic aldehyde," European Heart Journal, vol. 32, no. 8, pp. 1025-1038, 2011.

[43] P.-P. Hao, L. Xue, X.-L. Wang et al., "Association between aldehyde dehydrogenase 2 genetic polymorphism and serum 
lipids or lipoproteins: a meta-analysis of seven East Asian populations," Atherosclerosis, vol. 212, no. 1, pp. 213-216, 2010.

[44] Y. Li, D. Zhang, W. Jin et al., "Mitochondrial aldehyde dehydrogenase-2 (ALDH2) Glu504Lys polymorphism contributes to the variation in efficacy of sublingual nitroglycerin," The Journal of Clinical Investigation, vol. 116, no. 2, pp. 506-511, 2006.

[45] H. Zhang, D.-X. Gong, Y.-J. Zhang, S.-J. Li, and S. Hu, "Effect of mitochondrial aldehyde dehydrogenase-2 genotype on cardioprotection in patients with congenital heart disease," European Heart Journal, vol. 33, no. 13, pp. 1606-1614, 2012.

[46] J. Endo, M. Sano, T. Katayama et al., "Metabolic remodeling induced by mitochondrial aldehyde stress stimulates tolerance to oxidative stress in the heart," Circulation Research, vol. 105, no. 11, pp. 1118-1127, 2009.

[47] S. Takagi, S. Baba, N. Iwai et al., "The aldehyde dehydrogenase 2 gene is a risk factor for hypertension in Japanese but does not alter the sensitivity to pressor effects of alcohol: the Suita study," Hypertension Research, vol. 24, no. 4, pp. 365-370, 2001.

[48] K. Amamoto, T. Okamura, S. Tamaki et al., "Epidemiologic study of the association of low-Km mitochondrial acetaldehyde dehydrogenase genotypes with blood pressure level and the prevalence of hypertension in a general population," Hypertension Research, vol. 25, no. 6, pp. 857-864, 2002.

[49] P. Hui, T. Nakayama, A. Morita et al., "Common single nucleotide polymorphisms in Japanese patients with essential hypertension: aldehyde dehydrogenase 2 gene as a risk factor independent of alcohol consumption," Hypertension Research, vol. 30, no. 7, pp. 585-592, 2007.

[50] M. Tsuchihashi-Makaya, M. Serizawa, K. Yanai et al., "Geneenvironmental interaction regarding alcohol-metabolizing enzymes in the Japanese general population," Hypertension Research, vol. 32, no. 3, pp. 207-213, 2009.

[51] Y. Wang, Y. Zhang, J. Zhang et al., "Association of a functional single-nucleotide polymorphism in the ALDH2 gene with essential hypertension depends on drinking behavior in a Chinese Han population," Journal of Human Hypertension, vol. 27, no. 3, pp. 181-186, 2013.

[52] M. Guivernau, E. Baraona, and C. S. Lieber, "Acute and chronic effects of ethanol and its metabolites on vascular production of prostacyclin in rats," Journal of Pharmacology and Experimental Therapeutics, vol. 240, no. 1, pp. 59-64, 1987.

[53] Y.-C. Chang, Y.-F. Chiu, I.-T. Lee et al., "Common ALDH2 genetic variants predict development of hypertension in the SAPPHIRe prospective cohort: gene-environmental interaction with alcohol consumption," BMC Cardiovascular Disorders, vol. 12, article 58, 2012.

[54] T. Nakagawa, A. Kajiwara, J. Saruwatari et al., "The combination of mitochondrial low enzyme-activity aldehyde dehydrogenase 2 allele and superoxide dismutase 2 genotypes increases the risk of hypertension in relation to alcohol consumption," Pharmacogenetics and Genomics, vol. 23, no. 1, pp. 34-37, 2013.

[55] O. Niemelä, "Distribution of ethanol-induced protein adducts in vivo: relationship to tissue injury," Free Radical Biology and Medicine, vol. 31, no. 12, pp. 1533-1538, 2001.

[56] Acetaldehyde, vol. 36 of IARC Monographs on the Evaluation of Carcinogenic Risks to Humans, IARC, 1985.

[57] H. K. Seitz, S. Matsuzaki, A. Yokoyama, N. Homann, S. Vakevainen, and X. D. Wang, "Alcohol and Cancer," Alcoholism: Clinical and Experimental Research, vol. 25, supplement s1, pp. 137S-143S, 2001.
[58] "Alcohol drinking. Biological data relevant to the evaluation of carcinogenic risk to humans," IARC Monographs on the Evaluation of Carcinogenic Risks to Humans, vol. 44, pp. 101-152, 1988.

[59] K. Matsuo, N. Hamajima, M. Shinoda et al., "Gene-environment interaction between an aldehyde dehydrogenase-2 (ALDH2) polymorphism and alcohol consumption for the risk of esophageal cancer," Carcinogenesis, vol. 22, no. 6, pp. 913-916, 2001.

[60] Y.-J. Chen, C. Chen, D.-C. Wu et al., "Interactive effects of lifetime alcohol consumption and alcohol and aldehyde dehydrogenase polymorphisms on esophageal cancer risks," International Journal of Cancer, vol. 119, no. 12, pp. 2827-2831, 2006.

[61] W. F. Bosron and T.-K. Li, "Genetic polymorphism of human liver alcohol and aldehyde dehydrogenases, and their relationship to alcohol metabolism and alcoholism," Hepatology, vol. 6, no. 3, pp. 502-510, 1986.

[62] Y. Yukawa, M. Muto, K. Hori et al., "Combination of $\mathrm{ADH}_{1}{ }^{*} 2 / \mathrm{ALDH}_{2}{ }^{*} 2$ polymorphisms alters acetaldehydederived DNA damage in the blood of Japanese alcoholics," Cancer Science, vol. 103, no. 9, pp. 1651-1655, 2012.

[63] A. Yokoyama, T. Muramatsu, T. Omori et al., "Alcohol and aldehyde dehydrogenase gene polymorphisms influence susceptibility to esophageal cancer in Japanese alcoholics," Alcoholism: Clinical and Experimental Research, vol. 23, no. 11, pp. 1705-1710, 1999.

[64] A. Yokoyama, E. Tsutsumi, H. Imazeki, Y. Suwa, C. Nakamura, and T. Yokoyama, "Contribution of the alcohol dehydrogenase$1 \mathrm{~B}$ genotype and oral microorganisms to high salivary acetaldehyde concentrations in Japanese alcoholic men," International Journal of Cancer, vol. 121, no. 5, pp. 1047-1054, 2007.

[65] K. Matsuo, I. Oze, S. Hosono et al., "The aldehyde dehydrogenase $2(A L D H 2)$ Glu504Lys polymorphism interacts with alcohol drinking in the risk of stomach cancer," Carcinogenesis, vol. 34, no. 7, pp. 1510-1515, 2013.

[66] H.-L. Wang, P.-Y. Zhou, P. Liu, and Y. Zhang, "ALDH2 and $\mathrm{ADH} 1$ genetic polymorphisms may contribute to the risk of gastric cancer: a meta-analysis," PLoS ONE, vol. 9, no. 3, Article ID e88779, 2014.

[67] X.-F. Guo, J. Wang, S.-J. Yu et al., "Meta-analysis of the ADH1B and ALDH2 polymorphisms and the risk of colorectal cancer in East Asians," Internal Medicine, vol. 52, no. 24, pp. 2693-2699, 2013.

[68] H. Zhao, K.-J. Liu, Z.-D. Lei, S.-L. Lei, and Y.-Q. Tian, "Metaanalysis of the aldehyde dehydrogenases-2 (ALDH2) Glu487Lys polymorphism and colorectal cancer risk," PLOS ONE, vol. 9, no. 2, Article ID e88656, 2014.

[69] D. Zhou, L. Xiao, Y. Zhang et al., "Genetic polymorphisms of $\mathrm{ALDH} 2$ and $\mathrm{ADH} 2$ are not associated with risk of hepatocellular carcinoma among East Asians," Tumor Biology, vol. 33, no. 3, pp. 841-846, 2012.

[70] J.-Y. Choi, J. Abel, T. Neuhaus et al., "Role of alcohol and genetic polymorphisms of CYP2E1 and ALDH2 in breast cancer development," Pharmacogenetics, vol. 13, no. 2, pp. 67-72, 2003.

[71] T. Kawase, K. Matsuo, A. Hiraki et al., "Interaction of the effects of alcohol drinking and polymorphisms in alcoholmetabolizing enzymes on the risk of female breast cancer in Japan," Journal of Epidemiology, vol. 19, no. 5, pp. 244-250, 2009.

[72] M. Goedert and M. G. Spillantini, "A century of Alzheimer's disease," Science, vol. 314, no. 5800, pp. 777-781, 2006. 
[73] H. W. Querfurth and F. M. LaFerla, "Alzheimer's disease," The New England Journal of Medicine, vol. 362, no. 4, pp. 329-344, 2010.

[74] Y. Zhao and B. Zhao, "Oxidative stress and the pathogenesis of Alzheimer's disease," Oxidative Medicine and Cellular Longevity, vol. 2013, Article ID 316523, 10 pages, 2013.

[75] L. M. Sayre, D. A. Zelasko, P. L. R. Harris, G. Perry, R. G. Salomon, and M. A. Smith, "4-hydroxynonenal-derived advanced lipid peroxidation end products are increased in Alzheimer's disease," Journal of Neurochemistry, vol. 68, no. 5, pp. 2092-2097, 1997.

[76] W. A. Pedersen, N. R. Cashman, and M. P. Mattson, “The lipid peroxidation product 4-hydroxynonenal impairs glutamate and glucose transport and choline acetyltransferase activity in NSC19 motor neuron cells," Experimental Neurology, vol. 155, no. 1, pp. 1-10, 1999.

[77] M. D. Neely, K. R. Sidell, D. G. Graham, and T. J. Montine, “The lipid peroxidation product 4-hydroxynonenal inhibits neurite outgrowth, disrupts neuronal microtubules, and modifies cellular tubulin," Journal of Neurochemistry, vol. 72, no. 6, pp. 23232333, 1999.

[78] A. Takeda, M. A. Smith, J. Avilá et al., "In Alzheimer's disease, heme oxygenase is coincident with Alz50, an epitope of $\tau$ induced by 4-hydroxy-2-nonenal modification," Journal of Neurochemistry, vol. 75, no. 3, pp. 1234-1241, 2000.

[79] Q. Liu, M. A. Smith, J. Avilá et al., "Alzheimer-specific epitopes of tau represent lipid peroxidation-induced conformations," Free Radical Biology and Medicine, vol. 38, no. 6, pp. 746-754, 2005.

[80] E. Tamagno, M. Parola, P. Bardini et al., " $\beta$-site APP cleaving enzyme up-regulation induced by 4-hydroxynonenal is mediated by stress-activated protein kinases pathways," Journal of Neurochemistry, vol. 92, no. 3, pp. 628-636, 2005.

[81] I. Ohsawa, K. Nishimaki, C. Yasuda, K. Kamino, and S. Ohta, "Deficiency in a mitochondrial aldehyde dehydrogenase increases vulnerability to oxidative stress in PC12 cells," Journal of Neurochemistry, vol. 84, no. 5, pp. 1110-1117, 2003.

[82] S. Ohta and I. Ohsawa, "Dysfunction of mitochondria and oxidative stress in the pathogenesis of Alzheimer's disease: on defects in the cytochrome c oxidase complex and aldehyde detoxification," Journal of Alzheimer's Disease, vol. 9, no. 2, pp. 155-166, 2006.

[83] K. S. Montine, S. J. Oison, V. Amarnath, W. O. Whetsell Jr., D. G. Graham, and T. J. Montine, "Immunohistochemical detection of 4-hydroxy-2-nonenal adducts in Alzheimer's disease is associated with inheritance of APOE4," The American Journal of Pathology, vol. 150, no. 2, pp. 437-443, 1997.

[84] W. A. Pedersen, S. L. Chan, and M. P. Mattson, "A mechanism for the neuroprotective effect of apolipoprotein E: isoformspecific modification by the lipid peroxidation product 4 hydroxynonenal," Journal of Neurochemistry, vol. 74, no. 4, pp. 1426-1433, 2000

[85] B. Wang, J. Wang, S. Zhou et al., "The association of mitochondrial aldehyde dehydrogenase gene $(A L D H 2)$ polymorphism with susceptibility to late-onset Alzheimer's disease in Chinese," Journal of the Neurological Sciences, vol. 268, no. 1-2, pp. 172-175, 2008.

[86] S. Zhou, Huriletemuer, J. Wang et al., "Absence of association on aldehyde dehydrogenase $2(A L D H 2)$ polymorphism with Mongolian Alzheimer patients," Neuroscience Letters, vol. 468, no. 3, pp. 312-315, 2010.
[87] P.-P. Hao, Y.-G. Chen, J.-L. Wang, X. Wang, and Y. Zhang, "Meta-analysis of aldehyde dehydrogenase 2 gene polymorphism and Alzheimer's disease in East Asians," Canadian Journal of Neurological Sciences, vol. 38, no. 3, pp. 500-506, 2011. 

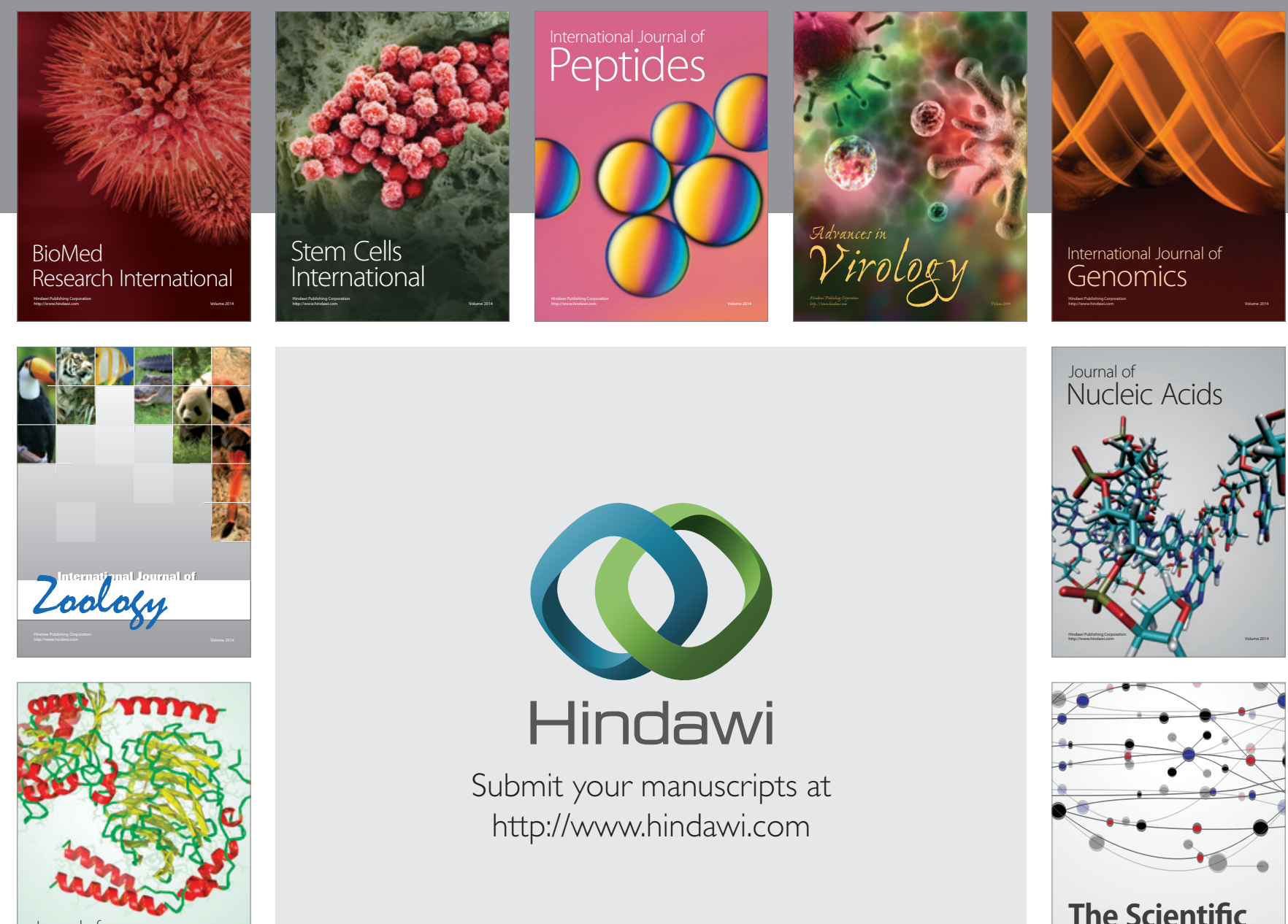

Submit your manuscripts at

http://www.hindawi.com

Journal of
Signal Transduction
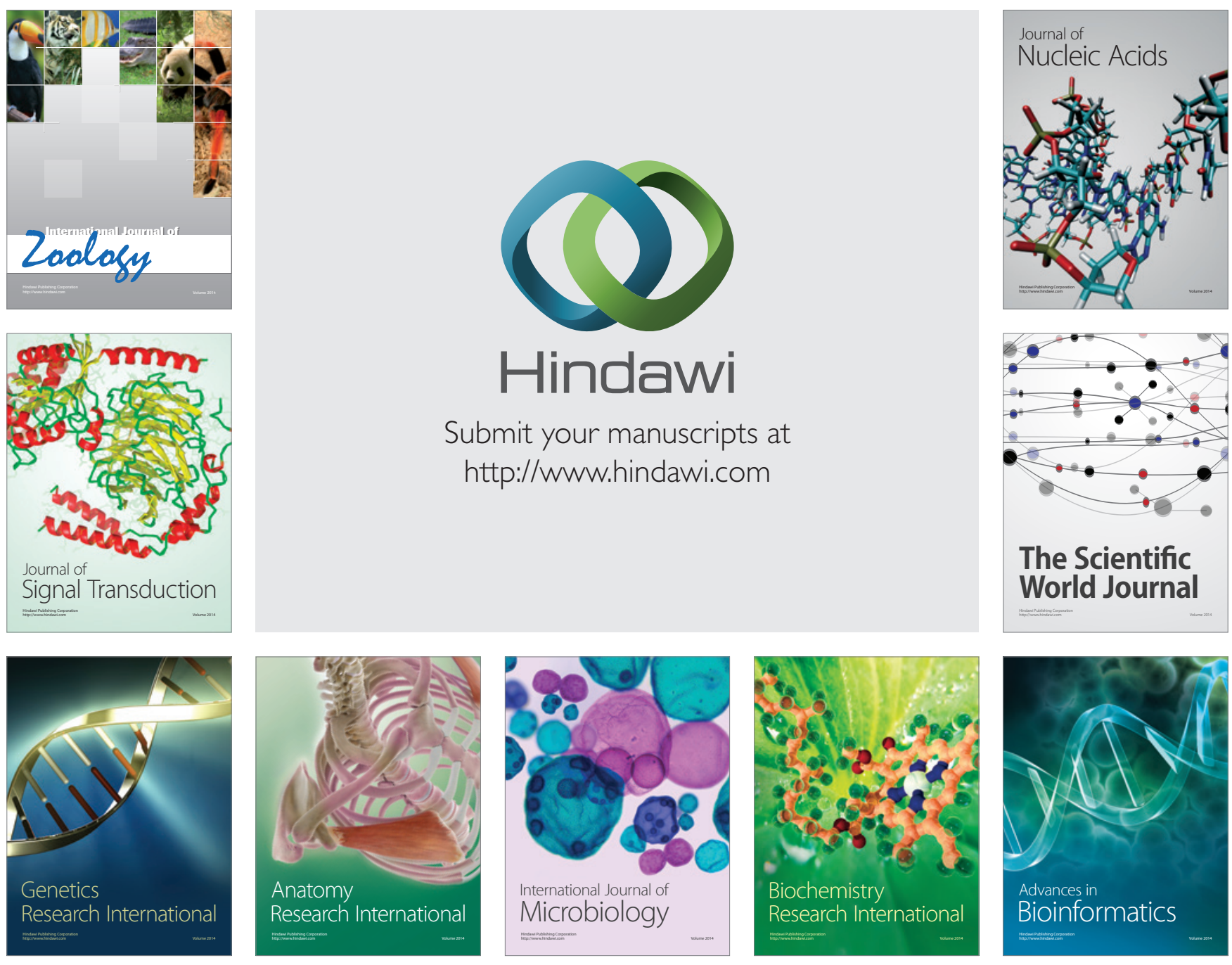

The Scientific World Journal
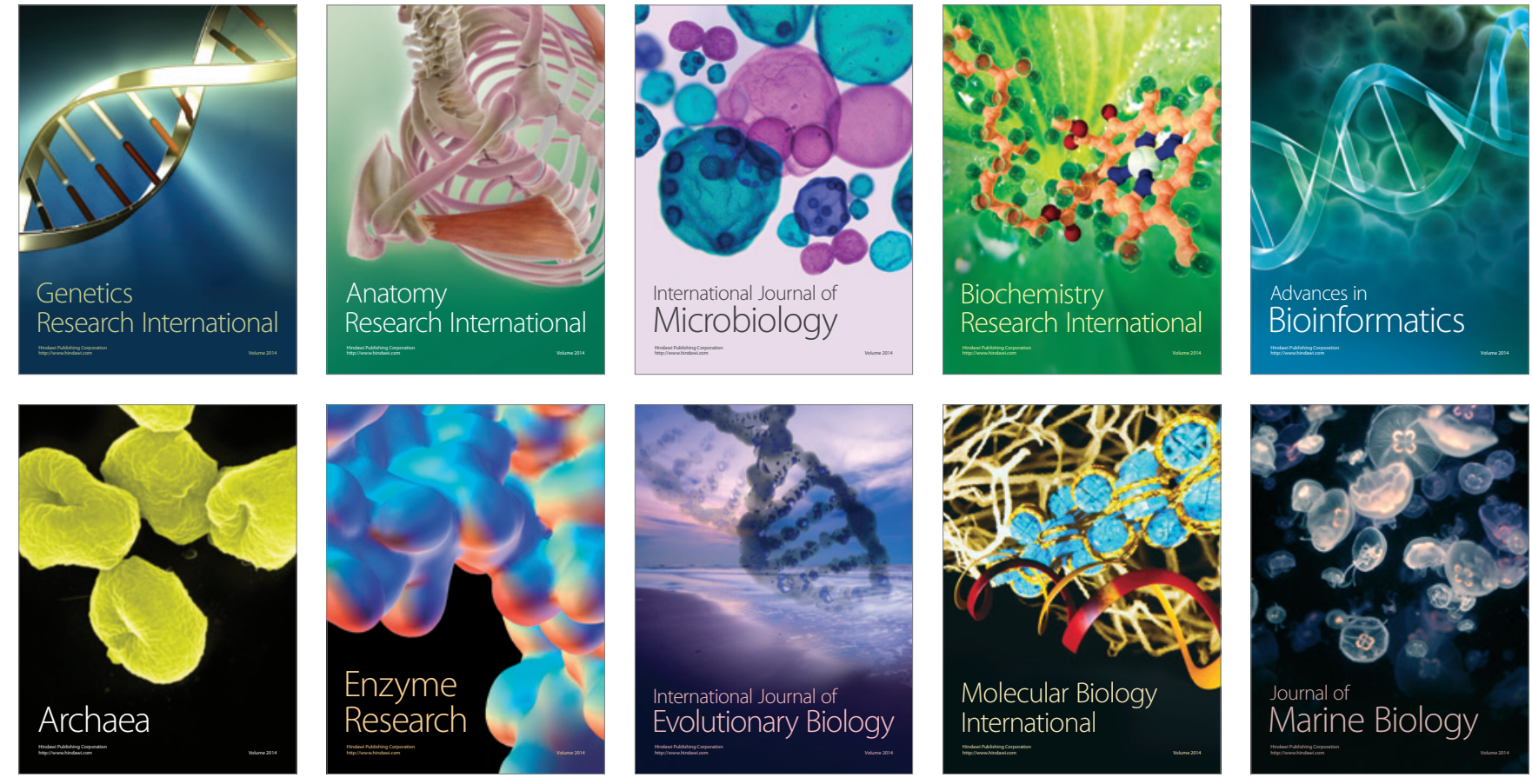